\title{
The envelope mass of red giant donors in type la supernova progenitors
}

\author{
$\mathrm{X}$. Meng and W. Yang \\ School of Physics and Chemistry, Henan Polytechnic University, Jiaozuo, 454000, PR China \\ e-mail: xiangcunmeng@hotmail.com
}

Received 1 November 2009 / Accepted 3 March 2010

\begin{abstract}
Context. The single degenerate model is the most widely accepted progenitor model of type Ia supernovae (SNe Ia), in which a carbon-oxygen white dwarf (CO WD) accretes hydrogen-rich material from a main sequence or a slightly evolved star (WD +MS) or from a red giant star (WD + RG), to increase its mass and explodes when approaching the Chandrasekhar mass. The explosion ejecta may impact the envelope of and strip off some hydrogen-rich material from the companion. The stripped-off hydrogen-rich material may manifest itself by means of a hydrogen line in the nebular spectra of SNe Ia. However, no hydrogen line is detected in the nebular spectra.

Aims. We compute the remaining amounts of hydrogen in red giant donors to see whether the conflict between theory and observations can be overcome.

Methods. By considering the mass-stripping effect from an optically thick wind and the effect of thermally unstable disk, we systematically carried out binary evolution calculation for WD + MS and WD + RG systems.

Results. Here, we focus on the evolution of WD + RG systems. We found that some donor stars at the time of the supernova explosion contain little hydrogen-rich material on top of the helium core (as low as $0.017 M_{\odot}$ ), which is smaller than the upper limit to the amount derived from observations of material stripped-off by explosion ejecta. Thus, no hydrogen line is expected in the nebular spectra of these SN Ia. We also derive the distributions of the envelope mass and the core mass of the companions from WD + RG channel at the moment of a supernova explosion by adopting a binary population synthesis approach. We rarely find a RG companion with a very low-mass envelope. Furthermore, our models imply that the remnant of the WD + RG channel emerging after the supernova explosion is a single low-mass white dwarf $\left(0.15-0.30 M_{\odot}\right)$.

Conclusions. The absence of a hydrogen line in nebular spectra of SNe Ia provides support to the proposal that the WD + RG system is the progenitor of SNe Ia.
\end{abstract}

Key words. white dwarfs - supernovae: general - binaries: general

\section{Introduction}

Although type Ia supernovae ( $\mathrm{SNe}$ Ia) are clearly important in determining cosmological parameters, e.g., $\Omega_{M}$ and $\Omega_{\Lambda}$ (Riess et al. 1998; Perlmutter et al. 1999), the progenitor systems of SNe Ia have not yet been confidently identified (Hillebrandt \& Niemeyer 2000; Leibundgut 2000). It is widely believed that a SN Ia is produced by the thermonuclear runaway of a carbonoxygen white dwarf (CO WD) in a binary system. The CO WD accretes material from its companion to increase its mass. When its mass reaches its maximum stable mass, it explodes as a thermonuclear runaway and almost half of the WD mass is converted into radioactive nickel-56 (Branch 2004). Two basic scenarios have been presented. One is the single degenerate (SD) model, which is widely accepted (Whelan \& Iben 1973; Nomoto et al. 1984). In this model, a CO WD increases its mass by accreting hydrogen- or helium-rich matter from its companion, and explodes when its mass approaches the Chandrasekhar mass limit. The companion may be a main-sequence star (WD+MS) or a red-giant star (WD+RG) (Yungelson et al. 1995; Li et al. 1997; Hachisu et al. 1999a,b; Nomoto et al. 1999, 2003; Langer et al. 2000; Han \& Podsiadlowski 2004; Chen \& Li 2007, 2009; Han 2008; Meng et al. 2009; Meng \& Yang 2010a; Lü 2009; Wang et al. 2009b, 2009c, 2010). The SD model has also been verified by many observations (see Meng \& Yang 2010b). An alternative is the theoretically less probable double degenerate (DD) model (Iben \& Tutukov 1984; Webbink 1984), in which a system of two CO WDs loses orbital angular momentum by means of gravitational wave radiation and finally merges. The merger remnant may explode if the total mass of the system exceeds the Chandrasekhar mass limit (see the reviews by Hillebrandt \& Niemeyer 2000; and Leibundgut 2000).

In the single degenerate model, the companion persists after the supernova explosion. The supernova ejecta collides with the envelope of and strips some hydrogen-rich material from the surface of the companion (Marietta et al. 2000; Meng et al. 2007; Pakmor et al. 2008). The stripped-off hydrogenrich material may manifest itself by means of narrow $\mathrm{H}_{\alpha}$ emission or absorption lines in later-time spectra of $\mathrm{SNe} \mathrm{Ia}$ (Chugai 1986; Filippenko 1997). The amount of the strippedoff material determines whether or not the narrow hydrogen line can be observed. Marietta et al. (2000) ran several highresolution two-dimensional numerical simulations of the collision between the ejecta and the companion. They claimed that about $0.15-0.17 M_{\odot}$ of hydrogen-rich material is stripped from a MS or a subgiant (SG) companion and $0.5 M_{\odot}$ from red giant (RG) star. Meng et al. (2007) used a simple analytic method but a more physical companion model than that used in 
Marietta et al. (2000) to simulate the interaction between $\mathrm{SNe}$ Ia ejecta and companions, and found that the minimum mass of the stripped-off material from a MS or SG star is $0.035 M_{\odot}$. However, the results of Marietta et al. (2000) and Meng et al. (2007) did not include confirmation by observations, i.e., no hydrogen line was detected in nebular spectra of some SNe Ia and the upper mass limit to the stripped-off material was set to be $0.02 M_{\odot}$ (Mattila et al. 2005; Leonard 2007). Pakmor et al. (2008) used a more physical companion model than and a similar numerical simulation to that of Marietta et al. (2000) to recalculate the interaction between the supernova ejecta and companion. They found results similar to those of Marietta et al. (2000). In certain circumstances, they claimed that these results agree with observations, and hence that theory does not conflict with observations. However, the special conditions envisaged by Pakmor et al. (2008) appear to be unrealistic according to detailed binary population synthesis results (Meng \& Yang 2010a). Based on the results of Pakmor et al. (2008), the amount of strippedoff material is between $0.06 M_{\odot}$ and $0.16 M_{\odot}$, which is consistent with the discovery of Marietta et al. (2000) and Meng et al. (2007; see also Meng \& Yang 2010a). The results of Pakmor et al. (2008) therefore do not resolve the confliction between theory and observations. Justham et al. (2009) proposed that the rotational effect of a CO WD may prevent its thermonuclear runaway until the accretion phase has ended, which could produce a RG companion with a low-mass envelope and reconcile theory and observations. We also suggest that failure to detect a hydrogen line in nebular spectra of some SNe Ia may imply that the $\mathrm{WD}+\mathrm{RG}$ channel is a means of producing SNe Ia. The amount of hydrogen-rich material obtained by Marietta et al. (2000) by means of WD + RG channel is higher than observed $\left(0.5 M_{\odot}\right)$, which may be due to the simplistic RG model used by Marietta et al. (2000).

In Sect. 2, we describe our binary evolution model. We present our evolutionary and binary population synthesis results in Sect. 3, and our discussions and conclusions in Sect. 4.

\section{Model and physics inputs}

Meng \& Yang (2010b) developed a comprehensive progenitor model for SNe Ia. In the model, the mass-stripping effect by optically thick wind (Hachisu et al. 1996) and the effect of a thermally unstable disk were included (Hachisu et al. 2008; Xu \& Li 2009). The prescription of Hachisu et al. (1999a) for WDs accreting hydrogen-rich material from their companions was applied to calculate the WD mass growth. The optically thick wind and the material stripped-off by the wind were assumed to remove the specific angular momentum of WD and its companion, respectively. In Meng \& Yang (2010b), both the WD + MS and WD + RG scenarios are considered, i.e., Roche lobe overflow (RLOF) begins at either the MS or RG stage. After the RLOF, WD accretes hydrogen-rich material from the donor and increases its mass smoothly. When the mass of the WD reaches $1.378 M_{\odot}$, the WD is assumed to explode as a SN Ia. The CO WDs may explode at the optically thick wind phase or after the optically thick wind while in either the stable or unstable hydrogen-burning phase or the dwarf nova phase. They considered more than 1600 different WD close binary evolution and obtained a dense model grid for SNe Ia. Based on the comprehensive model, Meng \& Yang (2010b) derived a Galactic birth rate of SNe Ia that is comparable to that inferred from observations. In the WD + MS channel obtained in Meng \& Yang (2010b), the companion models are similar to those in Meng et al. (2009). The masses stripped-off should then be similar to

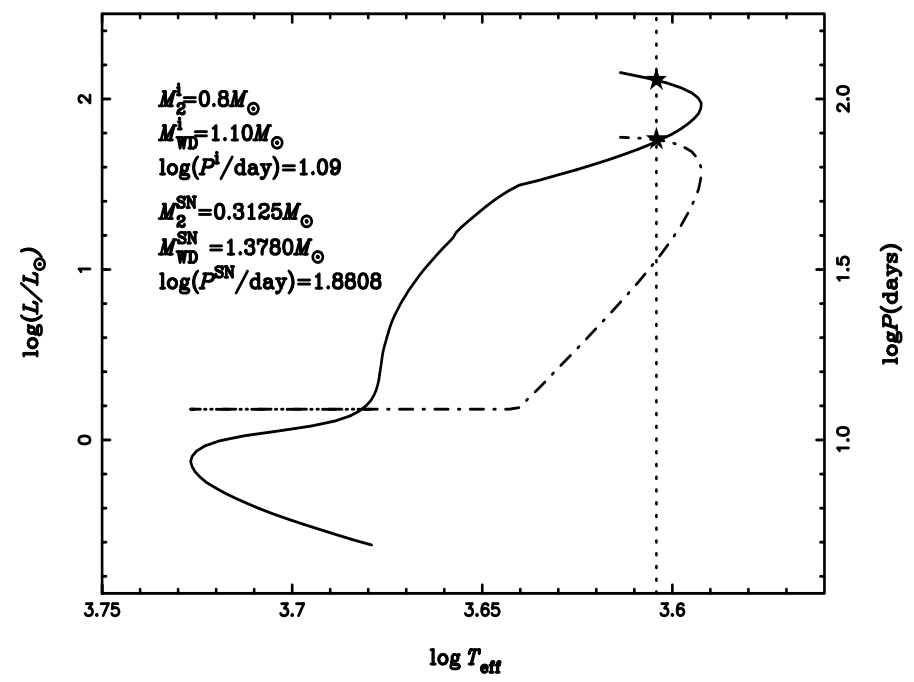

Fig. 1. An example of binary evolution calculations. The evolutionary track of the donor star in HRD is shown as solid curve and the evolution of orbital period is shown as dot-dashed curve. Dotted vertical line and asterisks indicate the position where the WD is assumed to explode as a SN Ia.

those found in Meng et al. (2007), i.e., higher than $0.035 M_{\odot}$. We therefore only considered the case of a WD + RG channel, which may only contribute to a fraction of all observed SNe Ia (see Meng \& Yang 2010b and Wang et al. 2010). All our methods for calculating the binary evolution and the physics inputs into the binary evolution calculation are similar to those in Meng \& Yang (2010b; see Meng \& Yang 2010b, for a detailed description of our method).

\section{Results}

\subsection{Binary evolution}

In Fig. 1, we show the evolutionary track of the donor star in the Hertzsprung-Russel diagram (HRD, solid line) and the evolution of the orbital period (dot-dashed line). The initial parameters of the binary system are also shown in the figure. The donor star evolves from the zero age main sequence (ZAMS). RLOF does not begin until the star enters the RG stage. The WD accretes hydrogen-rich material from the donor and increases its mass to $M_{\mathrm{WD}}^{\mathrm{SN}}=1.378 M_{\odot}$, where a SN Ia is assumed to occur, and $M_{2}^{\mathrm{SN}}=0.3125 M_{\odot}$ and $\log \left(P^{\mathrm{SN}} / \mathrm{day}\right)=1.8808$. At this point, the donor star consists of a helium core of $0.2955 M_{\odot}$ and a very thin hydrogen envelope of $0.017 M_{\odot}$; (we refer to Fig. 2, which shows the evolution of donor mass, core mass, and hydrogen envelope mass, where the definition of the core is identical to that in Han et al. 1994; and Meng et al. 2008). The envelope is so thin that the donor star has even left the RG and evolved to become a WD. Supernova ejecta impacts and strips off hydrogen-rich material from the envelope. Almost all the envelope material is stripped off (Marietta et al. 2000). The amount of the mass stripped-off from the companion should then be lower than $0.017 M_{\odot}$, which is lower than the upper mass limit of $0.02 M_{\odot}$ obtained from observations (Mattila et al. 2005; Leonard 2007). Therefore, no hydrogen line should be observed in the nebular spectra of this SN Ia, and our model can be reconciled with observations. This result may imply that the lack of detection of a hydrogen line in 


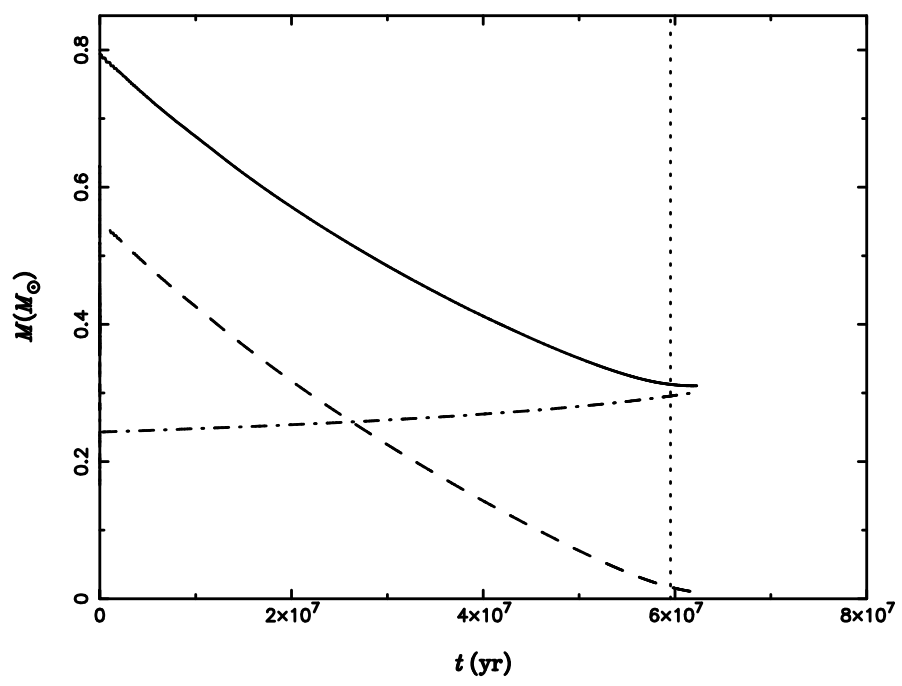

Fig. 2. The evolution of secondary mass (sold line), core mass (dotdashed line) and the hydrogen envelope mass (dashed line). Dotted vertical line indicates the position where the WD is expected to explode as a SN Ia. The zero point of time is set at the onset of RLOF.

nebular spectra of some SNe Ia could be evidence that the WD $+\mathrm{RG}$ channel represents a way of producing SNe Ia.

The RG model used by Marietta et al. (2000) consists of a helium core of $0.42 M_{\odot}$ and a thick envelope of $0.56 M_{\odot}$. The RG model may deviate from reality because the model was not obtained from a detailed binary evolution calculation and mass transfer between CO WD and the RG star was not considered. If mass transfer had been considered, the structure of the companion would differ significantly from that used in Marietta et al. (2000, see Sects. 3.2 and 4.3).

\subsection{Binary population synthesis}

To obtain the distributions of the envelope mass and the core mass of companion stars from WD + RG channel at the moment of SN explosion, we performed a detailed Monte Carlo simulation using Hurley's rapid binary evolution code (Hurley et al. $2000,2002)$. In the simulation, if a binary system evolves to a $\mathrm{WD}+\mathrm{RG}$ stage, and the system is located in the $\left(\log P^{\mathrm{i}}, M_{2}^{\mathrm{i}}\right)$ plane for SNe Ia at the onset of RLOF, we assume that an $\mathrm{SN}$ Ia is then produced. The envelope mass and the core mass of the WD binary at the moment of SN explosion are obtained by interpolation in the three-dimensional grid $\left(M_{\mathrm{WD}}^{\mathrm{i}}, M_{2}^{\mathrm{i}}, \log P^{\mathrm{i}}\right)$ obtained in Meng \& Yang (2010b).

In the simulation, we follow the evolution of 40 million sample binaries. The evolutionary channel was described in Meng \& Yang (2010b). As for Meng \& Yang (2010b), we adopted the following input to the simulation: (1) a constant star formation rate (SFR)over the past $15 \mathrm{Gyr}$; (2) the initial mass function (IMF) of Miller \& Scalo (1979); (3) the mass-ratio distribution is constant; (4) the distribution of separations is constant in $\log a$ for wide binaries, where $a$ is the orbital separation; (5) a circular orbit is assumed for all binaries; (6) the common envelope (CE) ejection efficiency $\alpha_{\mathrm{CE}}$, which denotes the fraction of the released orbital energy used to eject the CE, is set to be either 1.0 or 3.0 (see Meng \& Yang (2010b) for details of the parameter input).

In Figs. 3 and 4, we show current-epoch-snapshot distributions of the envelope mass and the core mass of companions produced by the WD + RG channel at the moment of supernova

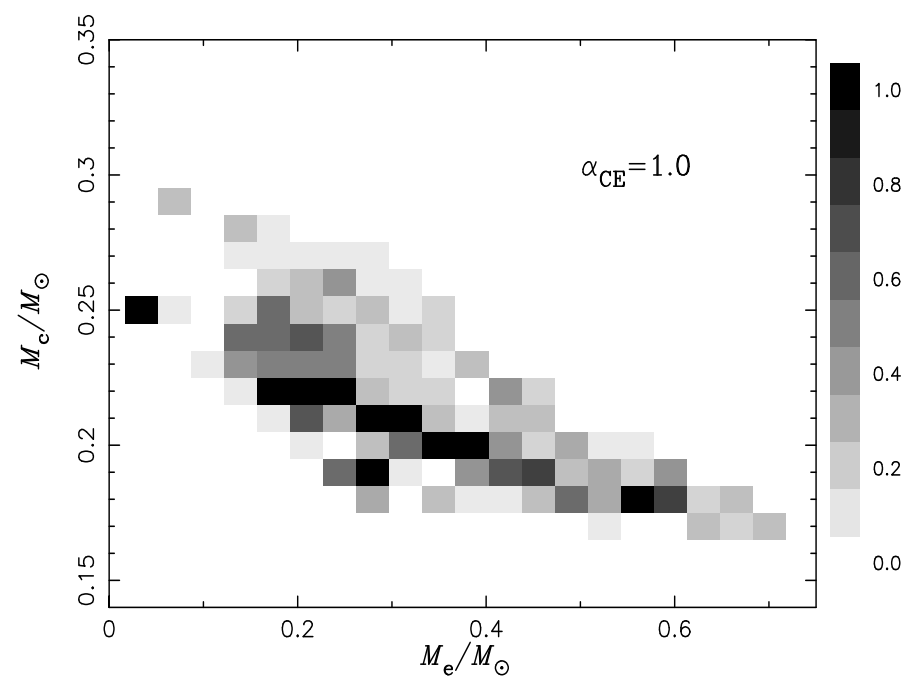

Fig. 3. Snapshot distribution of the envelope mass $M_{\mathrm{e}}$ and the core mass $M_{\mathrm{c}}$ of companion stars from WD $+\mathrm{RG}$ channel at current epoch with $\alpha_{\mathrm{CE}}=1.0$.

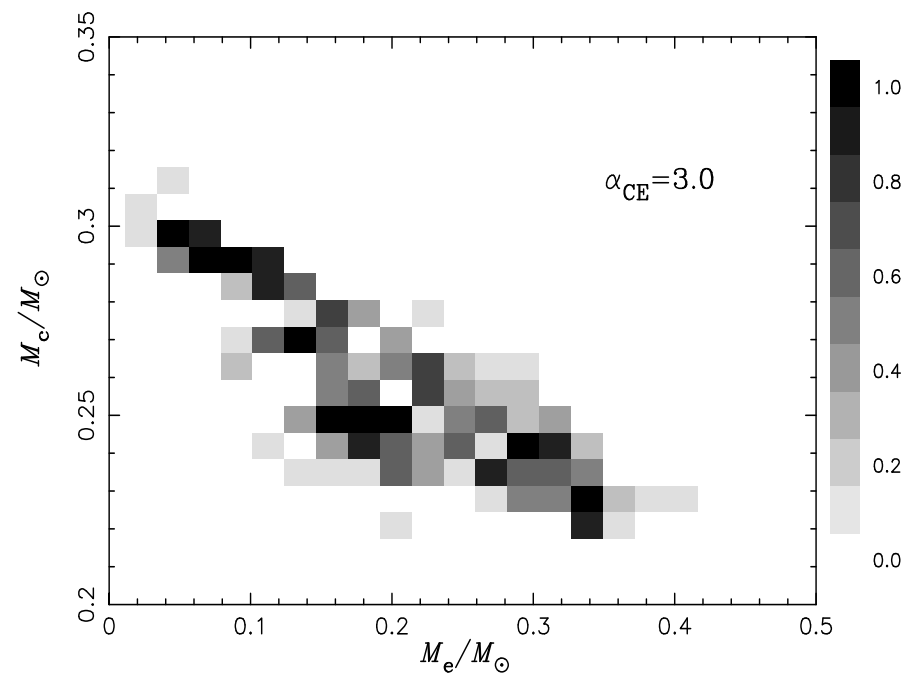

Fig. 4. Similar to Fig. 3 but for $\alpha_{\mathrm{CE}}=3.0$.

explosion with different $\alpha_{\mathrm{CE}}{ }^{1}$. In these figures, we can discern a clear trend, i.e., that core mass decreases in general with envelope mass. This is a natural consequence of stellar evolution. In addition, varying $\alpha_{\mathrm{CE}}$ may have a significant effect on the distributions. For $\alpha_{\mathrm{CE}}=1.0$, the envelope mass of companions is mainly between $0.1 M_{\odot}$ and $0.6 M_{\odot}$, while it is lower than $0.4 M_{\odot}$ for $\alpha_{\mathrm{CE}}=3.0$. This is because that for a given primordial binary system, high $\alpha_{\mathrm{CE}}$ is indicative of a longer orbital period after CE ejection. When the RG star fills its Roche lobe, the star then consists of a low-mass envelope and a high-mass core. As a result, the lower mass limit of the core mass for $\alpha_{\mathrm{CE}}=3.0$ is higher than that for $\alpha_{\mathrm{CE}}=1.0$, while the upper mass limit of the envelope mass for $\alpha_{\mathrm{CE}}=3.0$ is lower than that for $\alpha_{\mathrm{CE}}=1.0$.

\footnotetext{
${ }^{1}$ In the figures, we do not show the cases of WD + MS because: (i) the definition of the core for a MS star is incorrect; (ii) the interaction between supernova ejecta and the companion is complex, i.e., the stripped-off mass from the companion strongly depends on both the evolutionary stage of the MS star at the onset of RLOF and the initial parameters of a WD + MS system (Meng et al. 2007); and (iii) the study of WD + MS is beyond the scope of this paper.
} 
The core mass is between $0.15 M_{\odot}$ and $0.3 M_{\odot}$. After the interaction between supernova ejecta and RG companion, the RG companion loses almost its entire envelope (96-98\%) leaving only the core of the star (Marietta et al. 2000). This may be a channel to forming single low-mass white dwarf (Justham et al. 2009).

However, there are only a few systems with $M_{\mathrm{e}}<0.02 M_{\odot}$ in our sample, which means that the binary sequence considered in this paper may only be able to explain some individual observations (Mattila et al. 2005; Leonard 2007).

\section{Discussions and conclusions}

\subsection{Age}

We have found that if a SN Ia originates from the WD + RG channel, the donor star may almost become a helium WD and only some hydrogen-rich material remains on top of the helium WD (as little as $0.017 M_{\odot}$ ), which means that the upper limit mass to the stripped-off from the companion by supernova ejecta is $0.017 M_{\odot}$. No hydrogen line is then expected in the nebular spectra of some SNe Ia and our results may explain the conflict between theory and observation, i.e, theory predicts that the stripped-off material should be greater than $0.035 M_{\odot}$ (Marietta et al. 2000; Meng et al. 2007; Meng \& Yang 2010b), while observations indicate that the upper mass limit of the stripped-off material is $0.02 M_{\odot}$ (Mattila et al. 2005; Leonard 2007). No hydrogen line has been detected in nebular spectra of some $\mathrm{SNe}$ Ia may indicate that the progenitors of the observed SNe Ia are from WD + RG systems. If the observed SNe Ia (SN 2001el, $2005 \mathrm{am}$, and $2005 \mathrm{cl}$ ) were produced by the WD + RG channel, they should originate in an old population. Unfortunately, there is no constraint on the age of the three SNe Ia. We checked the types of their host galaxies and found that apart from the host galaxy of SN 2005cl (MCG-01-39-003), which is a S0 galaxy (Wang et al. 2009a; Bufano et al. 2009), they are both spiral galaxies, i.e. the host galaxy of SN 2001el (NGC 1448) is a Scd galaxy (Wang et al. 2003, 2006) and the host galaxy of SN 2001 am (NGC 2811) is a Sa galaxy (Bufano et al. 2009). The progenitor of SN $2005 \mathrm{cl}$ may therefore belong to an old population, but we are unable to infer any information about the population of the other SNe Ia. However, we note that all three SNe Ia are located at the edge of their host galaxy and that SN $2005 \mathrm{cl}$ is even located in a tail extending from MCG-01-39-003. Is this phenomenon evidence of an old population? It is possible because halo stars in general belong to an old population. All three $\mathrm{SNe}$ Ia are located at the edge of their host galaxy may be an observational select effect because we are more likely to observe a SN Ia at the outskirts of a host galaxy rather than its inner part. This selection effect might increase the probability that a SN Ia is produced by the WD $+\mathrm{RG}$ channel with a low-mass envelope being observed.

\section{2. $W D+R G$ system}

Relative to that of the WD + MS system, the Galactic birth rate of the WD + RG channel is low (see Meng \& Yang 2010b and Wang et al. 2010). However, since the Galactic birth rate of $\mathrm{SNe}$ Ia predicted by the model in Meng \& Yang (2010b) is lower than that inferred from observations, the WD + RG channel should be carefully investigated because the progenitors of some $\mathrm{SNe}$ Ia (e.g. SN 2006X and SN 2007on) are possible WD + RG systems (Patat et al. 2007a; Voss \& Nelemans 2008). In addition, some recurrent nova (belonging to $\mathrm{WD}+\mathrm{RG}$ ) are suggested to be the candidates of SNe Ia progenitors (Hachisu et al. 1999b; Hachisu \& Kato 2006; Hachisu et al. 2007). In this paper, we even proposed that the prevalence of the WD + RG channel is why no hydrogen line was detected in nebular spectra of some SNe Ia, although the probability of its occurrence is low.

\subsection{Interaction between supernova ejecta and companion}

Marietta et al. (2000) performed several high-resolution twodimensional numerical simulations of the collision between the supernova ejecta and companion, and found that a red-giant donor will lose almost its entire envelope (96-98\%) due to the impact leaving only the core of the star $\left(\simeq 0.42 M_{\odot}\right)$. The RG star used in Marietta et al. (2000) consists of a helium core of $0.42 M_{\odot}$ and an envelope of $0.56 M_{\odot}$, which are not comparable to those obtained by our simulations (see Figs. 3 and 4). In addition, the radius of their $\mathrm{RG}$ model is $180 R_{\odot}$, which corresponds to an orbital period of $\sim 900$ days. Too long to compare with our simulation (see Fig. 1), the orbital period leads to a lower envelope binding energy than produced by the model developed in this paper since the binding energy of the envelope is determined mainly by the radius of the RG star (Meng et al. 2008). The envelope of the RG model used by Marietta et al. (2000) is then more likely to be stripped off and the amount of material stripped-off by the RG companion in Marietta et al. (2000) might be overestimated. A more detailed numerical simulation of the interaction between supernova ejecta and an RG companion should therefore be performed by a more physical companion model than that in Marietta et al. (2000).

\subsection{Alternative explanation of the lack of hydrogen}

The absence of hydrogen lines in the nebular spectra of SNe Ia may have other explanations. The RG companion with a small hydrogen-rich envelope may be the results of either a fine-tuning effect as suggested in this paper, or a physical process. For example, Justham et al. (2009) suggested that a rotational effect of WD could prevent the thermonuclear runaway occurring until the accretion phase has ended, which could also produce a RG companion with a low-mass envelope. Rotation may also increase the probability of a SN Ia from WD + RG channel with low-mass envelope being observed. An alternative explanation of the lack of hydrogen is that the amount of stripped-off material might has been dramatically overestimated as discussed above.

Based on the discussions above, further attempts to observe hydrogen lines in nebular spectra of SNe Ia are encouraged to check our suggestion. The WD + RG system may also be an origin of single low-mass white dwarfs.

Acknowledgements. We are grateful to the anonymous referee for his/her constructive suggestions improving this manuscript greatly. This work was supported by Natural Science Foundation of China under grant No. 10963001.

\section{References}

Branch, D. 2004, Nature, 431, 1044

Bufano, F., Immler, S., Turatto, M., et al. 2009, ApJ, 700, 1456

Chen, W., \& Li, X. 2007, ApJ, 658, L51

Chen, W., \& Li, X. 2009, ApJ, 702, 686

Chugai, N. N. 1986, SvA, 30, 563

Filippenko, A. V. 1997, ARA\&A, 35, 309

Hachisu, I., \& Kato, M. 2006, ApJ, 651, L141

Hachisu, I., Kato, M., \& Nomoto, K. 1996, ApJ, 470, L97

Hachisu, I., Kato, M., Nomoto, K. et al. 1999a, ApJ, 519, 314 
X. Meng and W. Yang: The envelope mass of red giant donors in type Ia supernova progenitors

Hachisu, I., Kato, M., \& Nomoto, K. 1999b, ApJ, 522, 487 Hachisu, I., Kato, M., Luna, \& G. J. M. 2007, ApJ, 659, L153 Hachisu, I., Kato, M., \& Nomoto, K. 2008, ApJ, 679, 1390 Han, Z. 2008, ApJ, 677, L109

Han, Z., \& Podsiadlowski, Ph. 2004, MNRAS, 350, 1301

Han, Z., Podsiadlowski, P., \& Eggleton, P. P. 1994, MNRAS, 270, 121

Hillebrandt, W., \& Niemeyer, J. C. 2000, ARA\&A, 38, 191

Hurley, J. R., Pols, O. R., \& Tout, C. A. 2000, MNRAS, 315, 543

Hurley, J. R., Tout, C. A., \& Pols, O. R. 2002, MNRAS, 329, 897

Iben, I., Jr., \& Tutukov, A. V. 1984, ApJS, 54, 335

Justham, S., Wolf, C., Podsiadlowski, P., \& Han, Z. 2009, A\&A, 493, 1081

Langer, N., Deutschmann, A., Wellstein, S., et al. 2000, A\&A, 362, 1046

Leibundgut, B. 2000, A\&AR, 10, 179

Leonard, D. C. 2007, ApJ, 670, 1275

Li, X. D., \& van den Heuvel, E. P. J. 1997, A\&A, 322, L9

Lü, G., Zhu, C., Wang, Z., \& Wang, N. 2009, MNRAS, 396, 1086

Marietta, E., Burrows, A., \& Fryxell, B. 2000, ApJS, 128, 615

Mattila, S., Lundqvist, P., Sollerman, J., et al. 2005, A\&A, 443, 649

Meng, X., \& Yang, W. 2010a, MNRAS, 401, 1118

Meng, X., \& Yang, W. 2010b, ApJ, 710, 1310

Meng, X., Chen, X., \& Han, Z. 2007, PASJ, 59, 835

Meng, X., Chen, X., \& Han, Z. 2008, A\&A, 487, 625

Meng, X., Chen, X., \& Han, Z. 2009, MNRAS, 395, 2103
Nomoto, K., Thielemann, F. -K., \& Yokoi, K. 1984, ApJ, 286, 644

Nomoto, K., Umeda, H., Hachisu, I., et al. 1999, in Type Ia Suppernova: Theory and Cosmology, ed. J. Truran, \& T. Niemeyer (New York: Cambridge Univ. Press), 63

Nomoto, K., Uenishi, T., Kobayashi, C., et al. 2003, in From Twilight to Highlight: The Physics of supernova, ed. W. Hillebrandt, \& B. Leibundgut, ESO/Springer serious, ESO Astrophysics Symposia (Berlin: Springer), 115

Pakmor, R., Röopke, F. K., Weiss, A., \& Hillebrandt, W. 2008, A\&A, 489, 943

Patat, F., Chandra, P., Chevalier, R., et al. 2007, Science, 317, 924 Perlmutter, S., Aldering, G., Goldhaber, G., et al. 1999, ApJ, 517, 565

Riess, A. G., Filippenko, A. V., Challis, P., et al. 1998, AJ, 116, 1009 Voss, R., \& Nelemans, G. 2008, Nature, 451, 802

Yungelson, L., Livio, M., Tutukov, A., et al. 1995, ApJ, 447, 656

Wang, L., Baade, D., Höflich P., et al. 2003, ApJ, 591, 1110

Wang, X., Wang, L., Pain, R., et al. 2006, ApJ, 645, 488

Wang, X., Li, W., Filippenko, A. V., et al. 2009a, ApJ, 697, 380

Wang, B., Meng, X., Chen, X., \& Han, Z. 2009b, MNRAS, 395, 847

Wang, B., Chen, X., Meng, X., \& Han, Z. 2009c, ApJ, 701, 1540

Wang, B., Li, X., \& Han, Z. 2010, MNRAS, 401, 2729

Webbink, R. F. 1984, ApJ, 277, 355

Whelan, J., \& Iben, I. 1973, ApJ, 186, 1007

Xu, X., \& Li, X. 2009, A\&A, 495, 243 